\title{
Éditorial
}

\section{Nécessaire retour du balancier}

Ce numéro thématique de la revue Organisations et territoires représente une première dans le genre. Peut-être pas une dernière. Il y a longtemps que nous jonglions avec le projet d'un numéro complet dédié à un territoire spécifique, notamment le "Saguenay historique » ou, en l'occurrence, la plus récente région administrative du Saguenay-Lac-Saint-Jean. Mais la convergence des astres ne s'y prêtait guère. Or, le lancement du mouvement Vision Saguenay 2025 en septembre 2003 a créé la conjoncture céleste tant attendue. Ayant comme vocation première la vulgarisation scientifique, la revue Organisations et territoires nous apparaissait alors comme un véhicule privilégié pour fertiliser la réflexion collective tant souhaitée. Un partenariat fut scellé.

Nous avons ainsi sollicité un groupe d'universitaires pour des contributions sur l'avenir de la région du Saguenay-Lac-Saint-Jean. L'objectif n'était pas tant de tous se livrer à un strict exercice méthodologique de prospective territoriale. Il s'agissait plutôt d'élaborer des projections futures sur la base de diverses analyses scientifiques régionales. Tous les auteurs n'ont pas répondu avec la même fidélité à cet appel pour l'utilisation d'une telle perspective. Mais tous les textes retenus par le comité éditorial concernent de plein fouet la région du Saguenay-Lac-Saint-Jean ou l'une de ses composantes internes, en ouvrant notamment sur le devenir de cette collectivité territoriale. La grande richesse des propos sélectionnés nous apparaît sans équivoque, notamment dans leur diversité et leur maturité. La pertinence sociale de ces textes proposés à votre lecture ne fait aussi aucun doute. Seront en ce sens fort bien servis les acteurs de la collectivité analysée dans leur recherche continue de nouvelles lumières. Évidemment, les auteurs ont aussi visé la pertinence académique de leurs propos. Car les étudiants désirent se familiariser bien sûr avec les théories et les concepts offerts par les sciences sociales, mais aussi avec des applications concrètes pour observer et analyser des phénomènes territoriaux réels. Ce numéro thématique devient alors un must.

Le mouvement Vision Saguenay 2025 frappe ainsi une balle à plusieurs buts dans son désir de mobilisation élargie autour d'une lecture renouvelée de l'avenir collectif. De nouveaux enjeux régionaux sont à relever, ne serait-ce que l'intégration de la nouvelle ville de Saguenay avec sa périphérie, la cruciale question démographique, le déficit démocratique, l'appropriation régionale de nouveaux leviers économiques, la création d'une classe ouvrière intermédiaire, les mécanismes relationnels pour structurer les filières de production. En réalité, la région du Saguenay-LacSaint-Jean doit innover plus que jamais dans ses modalités de gouvernance, alors que la sédimentation institutionnelle est réellement devenue une contrainte. À cet effet d'impérative innovation régionale, il est important de préciser que la composante méthodologique concernée par la «vision» fut insuffisamment renouvelée par les derniers exercices de la planification régionale. Voici pourquoi.

On sait que la planification appliquée à une région comme le Saguenay-Lac-Saint-Jean comporte quatre composantes distinctes mais interreliées (voir schéma), à exercer par une méthode ou une procédure appropriée. Il s'agit de la vision, du cadre stratégique, de la prise de décisions et de l'interaction entre les décideurs. Si la procédure de planification régionale mise en œuvre conduit à un équilibre fertile entre ces quatre composantes bien exercées, l'innovation sociale, culturelle, institutionnelle et économique sera maximisée en conséquence par l'entremise d'une liaison fructueuse entre la connaissance et l'action.

Or, dans la région administrative du Saguenay-LacSaint-Jean comme dans les autres régions du Québec depuis le début des années 1990, le cadre stratégique a hélas considérablement dominé les autres composantes de la planification par l'entremise de la mise en œuvre de ladite planification stratégique. L'envoûte- 
ment des décideurs régionaux à l'égard de l'encadrement, par les stratégies, de la dynamique régionale s'explique largement par le désir de compenser les faibles moyens financiers et réglementaires mis à leur disposition. Nul doute aussi que la volonté gouvernementale d'allouer les ressources publiques à l'aide d'ententes-cadres Québec-régions a aussi plaidé en faveur de cette planification stratégique. Il demeure néanmoins que cette préséance méthodologique du cadre stratégique affecte l'équilibre entre les composantes de la planification en éloignant les décideurs régionaux de l'optimum pleinement innovateur qui se situe au centre du schéma. Tant et si

\section{Les composantes de la planification territoriale}

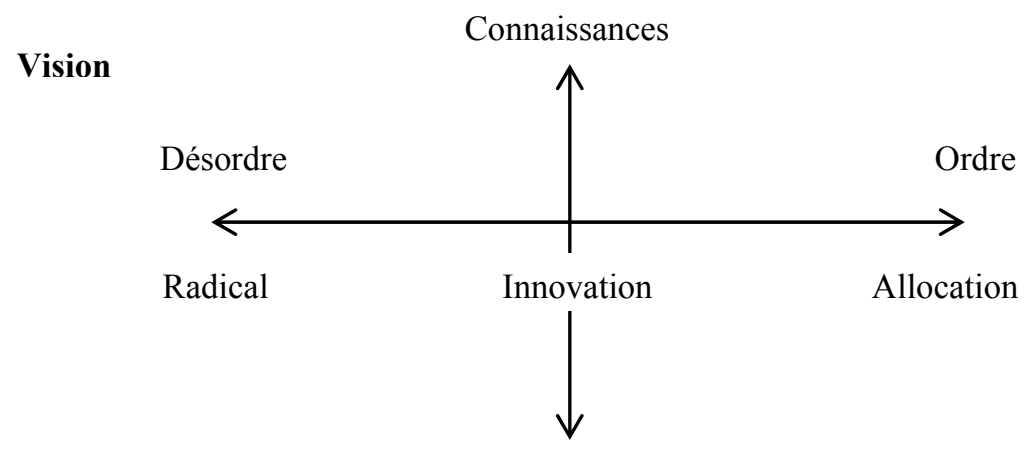

Interaction

Actions simple : stimuler l'interaction et la réflexion collective dans un esprit de fertilisation d'une vision régionale enrichie. En ce sens, le numéro thématique de la revue Organisations et territoires que vous avez entre les mains représente une modeste mais pertinente contribution vers une nouvelle procédure de planification territoriale vivement souhaitée par Vision Saguenay 2025. bien que les retombées issues de la planification stratégique régionale exercée au Québec durant la décennie 1990 n'ont pas illustré toute l'originalité et l'innovation de celles générées par la planification interactive exercée pendant les années 1980. Alors que les besoins étaient si importants. Les actions structurantes tant réclamées par les stratèges régionaux furent certes au rendez-vous, mais selon un volume beaucoup trop limité. En référence au schéma ci-dessus, la planification stratégique régionale génère trop d'ordre et insuffisamment de désordre pour s'avérer pleinement innovatrice ${ }^{1}$. Un retour du balancier vers un meilleur équilibre nous apparaît impératif. Et nous le recommandons vivement au législateur québécois.

À cet effet, le mouvement Vision Saguenay 2025 expérimente actuellement une nouvelle approche de nature prospective. La mobilisation effectuée provoque certes un effet de "décristallisation institutionnelle » dans le milieu. Le but du mouvement est fort tiples facettes de la réalité régionale complexe. Ce numéro représente ainsi non seulement un ouvrage de référence à placer dans le rayon Saguenay-Lac-SaintJean de votre bibliothèque, mais aussi une pièce à conviction ayant le double attribut de valeur scientifique et d'accessibilité à un large public qu'est devenue la grande famille des acteurs locaux et régionaux. Il devient ainsi un jalon incontournable dans le cumul de connaissances à propos du Saguenay-Lac-Saint-Jean et de son devenir.

\section{Note}

1 Voir le texte de M.-U. Proulx (1996). « Trois décennies de planification régionale au Québec », dans M.-U. Proulx, Le phénomène régional au Québec, Presses de l'Université du Québec.

Marc-Vrbain Proulx

Le Directeur 